ISSN 1112-9867

\title{
STIFFNESS MODULUS AND CREEP PROPERTIES OF THE COCONUT SHELL IN AN ASPHALT MIXTURE
}

\author{
H. Yaacob ${ }^{1}$, R. P. Jaya ${ }^{1, *}$, A. H. Madzaili ${ }^{1}$, N. A. $\operatorname{Hassan}^{1}$, M. E. Abdullah ${ }^{2}$ and D. S. Jayanti ${ }^{3}$ \\ ${ }^{1}$ Faculty of Civil Engineering, Department of Geotechnics and Transportation, \\ UniversitiTeknologi Malaysia, 81310 Johor Bahru, Malaysia \\ ${ }^{2}$ Faculty of Civil and Environmental Engineering, UniversitiTun Hussein Onn Malaysia, \\ 86400 BatuPahat, Johor, Malaysia \\ ${ }^{3}$ Faculty of Agricultural, Syiah Kuala of University, 23111 Banda Aceh, Indonesia
}

Published online: 10 November 2017

\begin{abstract}
Coconut shell (CS) is an agricultural waste engineered into a road construction material. This study was conducted to evaluate the stiffness modulus and dynamic creep properties of the asphaltic concrete containing CS as an aggregate replacement. A mixture design incorporating the bitumen penetration grade 60/70 was used to produce specimens for testing. Four CS replacements namely $0 \%, 10 \%, 20 \%, 30 \%$ and $40 \%$ by weight volumes were used. The asphaltic concrete performances were characterized by stiffness modulus, dynamic creep and the correlation between the strain and the loading time. Test results revealed that using CSs as aggregate replacements in the asphaltic concrete help increase the resilient modulus and creep stiffness at approximately $15 \%$. Therefore, the incorporation of CS exhibits considerable potential as road construction material.
\end{abstract}

Keywords: asphalt mixture; coconut shell; creep modulus; stiffness modulus; properties.

Author Correspondence, e-mail: ramadhansyah@utm.my

doi: http://dx.doi.org/10.4314/jfas.v9i6s.5 


\section{INTRODUCTION}

Conventional aggregate sources in Malaysia are depleting fast due to rapid growth of construction activities [1]. Currently, the transportation industry is under pressure to use alternative materials because of high consumption of aggregates in road construction [2-3]. These materials should be used wisely to attain sustainable development. A large number of studies have been conducted to search for a possible material as an aggregate replacement in road conductions [4-5]. Currently, using agricultural waste materials as an aggregate replacement becomes a trend in the construction industry [6-7]. The use of agricultural waste aims to minimize the production of waste, preserve the environment and improve the pavement durability [8-9]. Durability refers to the capability of the mixture to resist abrasion of the surface due to the high void content, which allows air and water to harden the asphalt prematurely. Coconut shell (CS) is an agricultural waste that can be used for durability resistance [10-11]. Several studies have been conducted using CS as coarse aggregates in lightweight concrete [12-13]. However, the use of CS in hot asphalt mixture has never been extensively studied. Therefore, the present study explored the effects of CS on the stiffness modulus and creep properties of an asphaltic concrete. This study focused on using the variation of CS from $0 \%$ to $40 \%$ as an aggregate replacement in the asphalt mixture.

\section{MATERIALS AND METHODS}

\subsection{Binder}

The bitumen used was a 60/70 PEN supplied by Chevron Malaysia. This grade bitumen is widely used in Malaysia and other countries. According to the specification of the manufacturer, the relative density value of the $60 / 70$ binder was 1.03 . The softening point and penetration were $52{ }^{\circ} \mathrm{C}$ and $65 \mathrm{dmm}$, respectively.

\subsection{Aggregate}

The granite aggregates supplied by Kuad Quarry Sdn. Bhd. were used throughout this study. Prior to aggregate batching, the aggregates were washed, dried, and sieved into their respective size ranges. The granite aggregate exhibited a specific gravity of $2.65 \mathrm{~g} / \mathrm{cm}^{3}$ and the ACV and water absorption were $26.1 \%$ and $0.48 \%$ correspondingly.

\subsection{Coconut Shell}


The CS size used as an aggregate replacement was $5 \mathrm{~mm}$. In addition, the replacement levels of CS were $0 \%, 10 \%, 20 \%, 30 \%$ and $40 \%$ by total weight. According to [14], the specific gravity for coarse CS is 1.48 and the water absorption is $29.3 \%$.

\subsection{Gradation}

The aggregate gradation conformed to the specifications for the asphaltic concrete AC14 [15]. The median gradation was selected as the target gradation as illustrated in Fig. 1 [15].

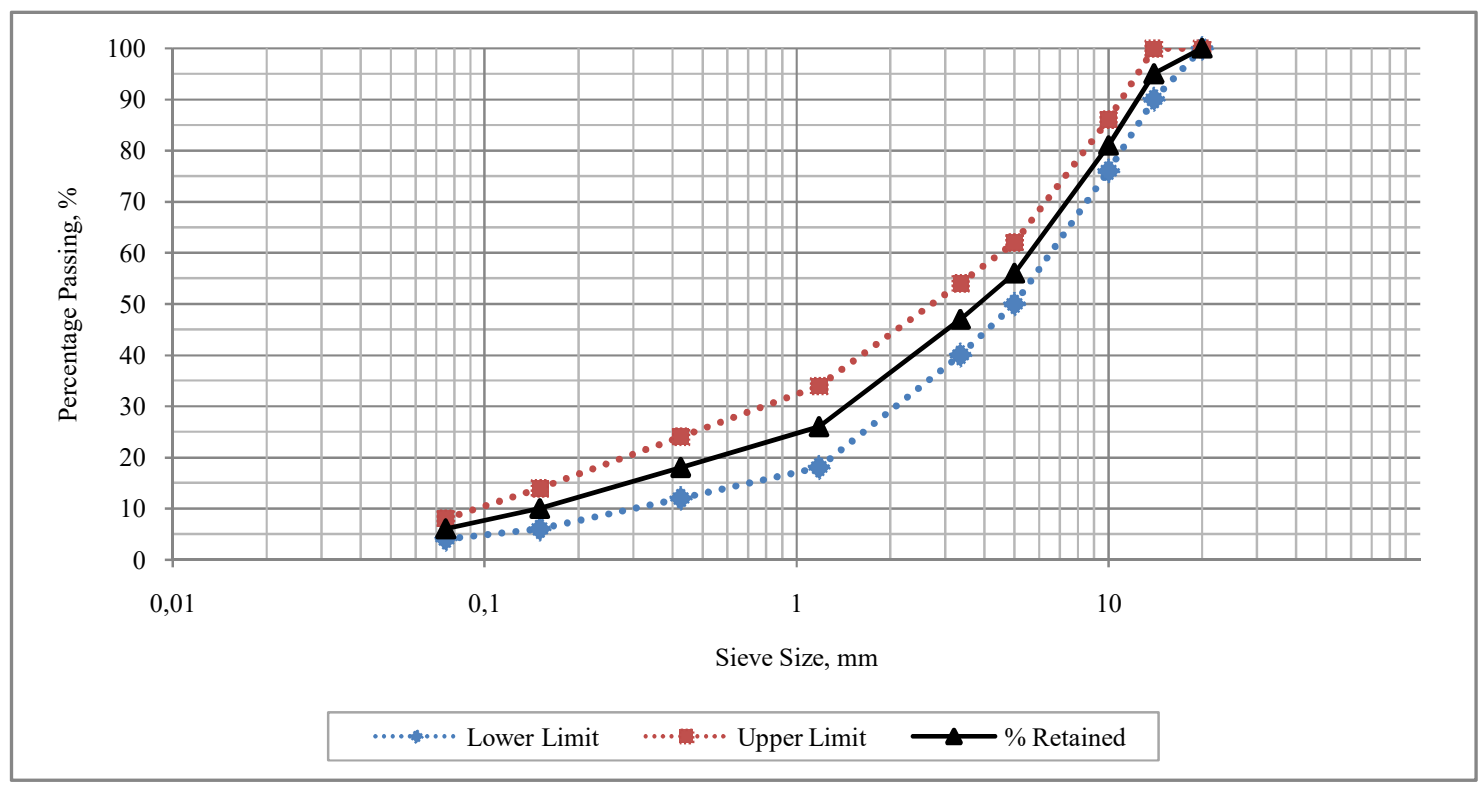

Fig.1. Gradation limit for asphaltic concrete

\subsection{Mixture Preparation}

An ordinary Portland cement and hydrated lime were used as fillers. The 60/70 PEN bitumen was used as a binder. First, the aggregates were mixed into batches according to the designated gradations and weight. These batches were heated in an oven at designated mixing temperature for at least $4 \mathrm{~h}$ prior to the mixing process. The heated aggregate batches were mixed with a specified amount of bitumen. The mixes were compacted with 75 blows [15] on each side with the standard Marshall hammer. After compaction, the specimens were removed from the molds and allowed to cool overnight.

\subsection{Resilient Modulus Test}

A five-pulse indirect tensile modulus test conforming to the [16] test method was conducted using the universal testing machine. The specimen was tested at $25^{\circ} \mathrm{C}$ after a $4 \mathrm{~h}$ conditioning period. In the test, a pulsed diametral loading force was applied to a specimen, and the 
resulting total recoverable diametral strain was measured. The strains in the same axes were not measured. A Poisson's ratio of 0.4 was assumed for the material. At a fixed level of applied peak force, the test sequence consisted of the application of 150 conditioning pulses, which were followed by five pulses, where data acquisition occurred. The test procedure was repeated by positioning the specimen at $90^{\circ}$.

\subsection{Dynamic Creep Test}

The dynamic creep test was developed to estimate the rutting potential of the asphalt mixes in accordance with the procedures outlined in [17]. This test applied 0.3 MPa repeated pulses of uniaxial stress parallel to the specimen with 3600 recovery cycles and measured the deformation in the same direction using the linear variable differential transducers. An actual dynamic creep test was conducted at $40^{\circ} \mathrm{C}, 1 \mathrm{~h}$ loading time and $0.1 \mathrm{MPa}$ applied stress.

\section{RESULTS AND DISCUSSION}

\subsection{Resilient Modulus}

The resilient modulus test results for the asphaltic concrete prepared with CS at varying percentages are illustrated in Fig. 2. Generally, the increment in CS content causes an increase in the resilient modulus to a CS content of $20 \%$. Thereafter, the resilient modulus starts to decrease. The resilient modulus is the modulus of elasticity when the asphalt sample is loaded within its elastic range, where the deformation is fully recoverable. Therefore, the specimens exhibiting high resilient modulus indicate less flexibility under loading. In this study, the $10 \%$ $\mathrm{CS}$ in the asphalt mixture responds with higher sensitivity to temperature changes compared with those of $0 \%, 20 \%, 30 \%$ and $40 \% \mathrm{CS}$. The resilient modulus of the asphaltic concrete at $25^{\circ} \mathrm{C}$ is also higher than that of $40^{\circ} \mathrm{C}$. The resilient modulus of the asphaltic concrete containing CS reduces by approximately $64 \%-80 \%$ when the test temperature increases from $25^{\circ} \mathrm{C}$ to $40^{\circ} \mathrm{C}$. For instance, the resilient modulus of $0 \% \mathrm{CS}$ mix reduces by $360 \mathrm{MPa}$ through an increment of $40^{\circ} \mathrm{C}$ in the test temperature, and the corresponding reduction for $10 \% \mathrm{CS}$ is $383 \mathrm{MPa}$. 


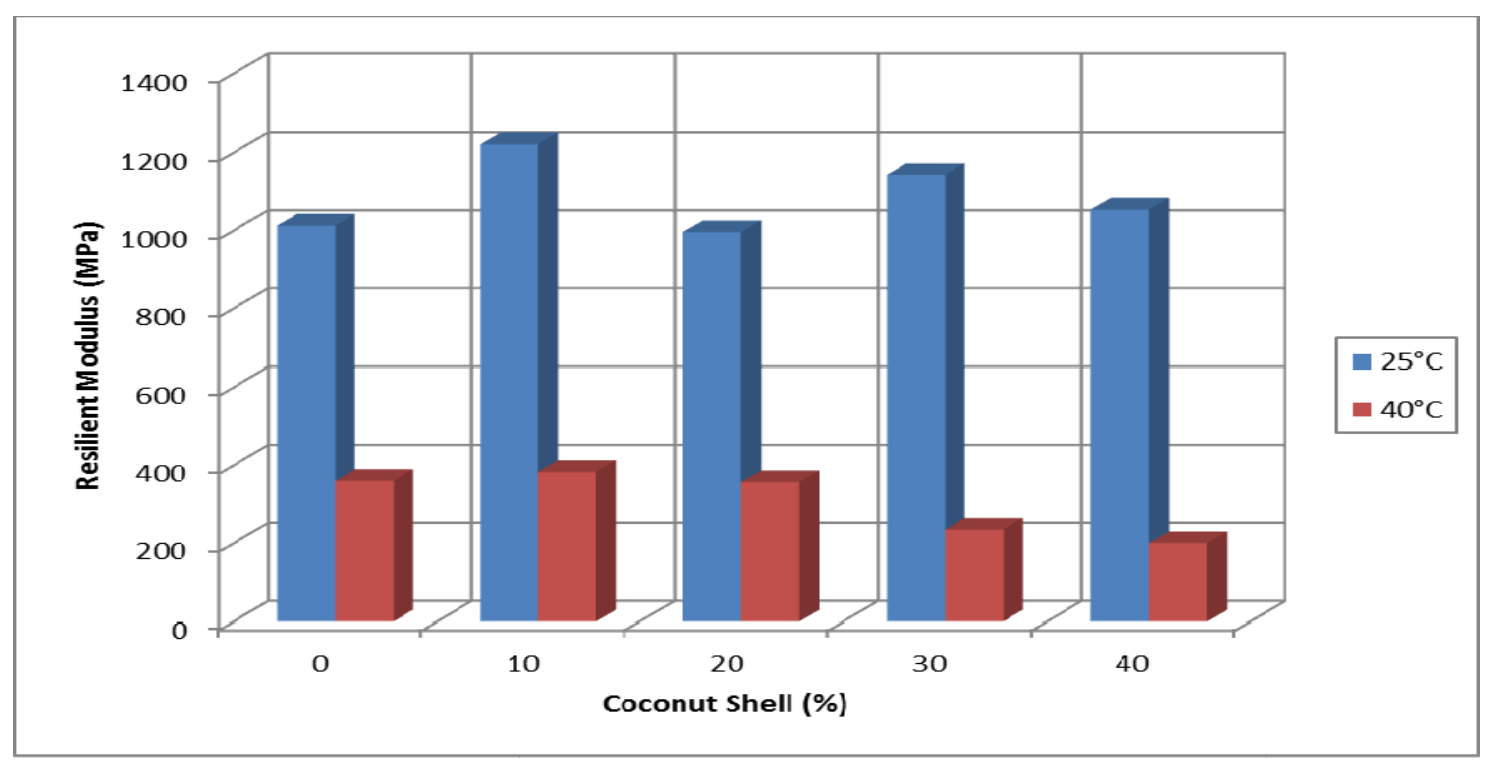

Fig.2. Resilient modulus of asphalt mixture at different percent CS

\subsection{Dynamic Creep}

The results of the dynamic creep test on CS mixes are presented graphically in Fig. 3. Each value on the figure is an average reading of the three samples tested. Generally, the mixes incorporating $\mathrm{CS}$ as aggregate replacement perform better than the mixes with virgin aggregate. The percentage increase in creep stiffness is remarkably pronounced with CS mixes. The increase in creep stiffness may be due to the superior interlocking property of aggregate-binder adhesion exhibited by the CS. For instance, the creep stiffness of $0 \% \mathrm{CS}$ mix is $14.7 \mathrm{MPa}$ but the stiffness modulus of the asphaltic concrete with $10 \% \mathrm{CS}$ mix is 15.5 $\mathrm{MPa}$, which represents a 5\% increase. Moreover, the dynamic creep increases with the increasing CS content to a peak level and then decreases with further additions. The $20 \%$ CS asphaltic concrete mix exhibits the highest creep stiffness compared with those of $0 \%, 10 \%$ and $30 \%$ CS. However, at $40 \%$ CS mix, the dynamic creep decreases to a value lower than that of $0 \%$ CS mix. Thus, the $20 \%$ replacement of CS with the original aggregate is considered the optimal limit. 


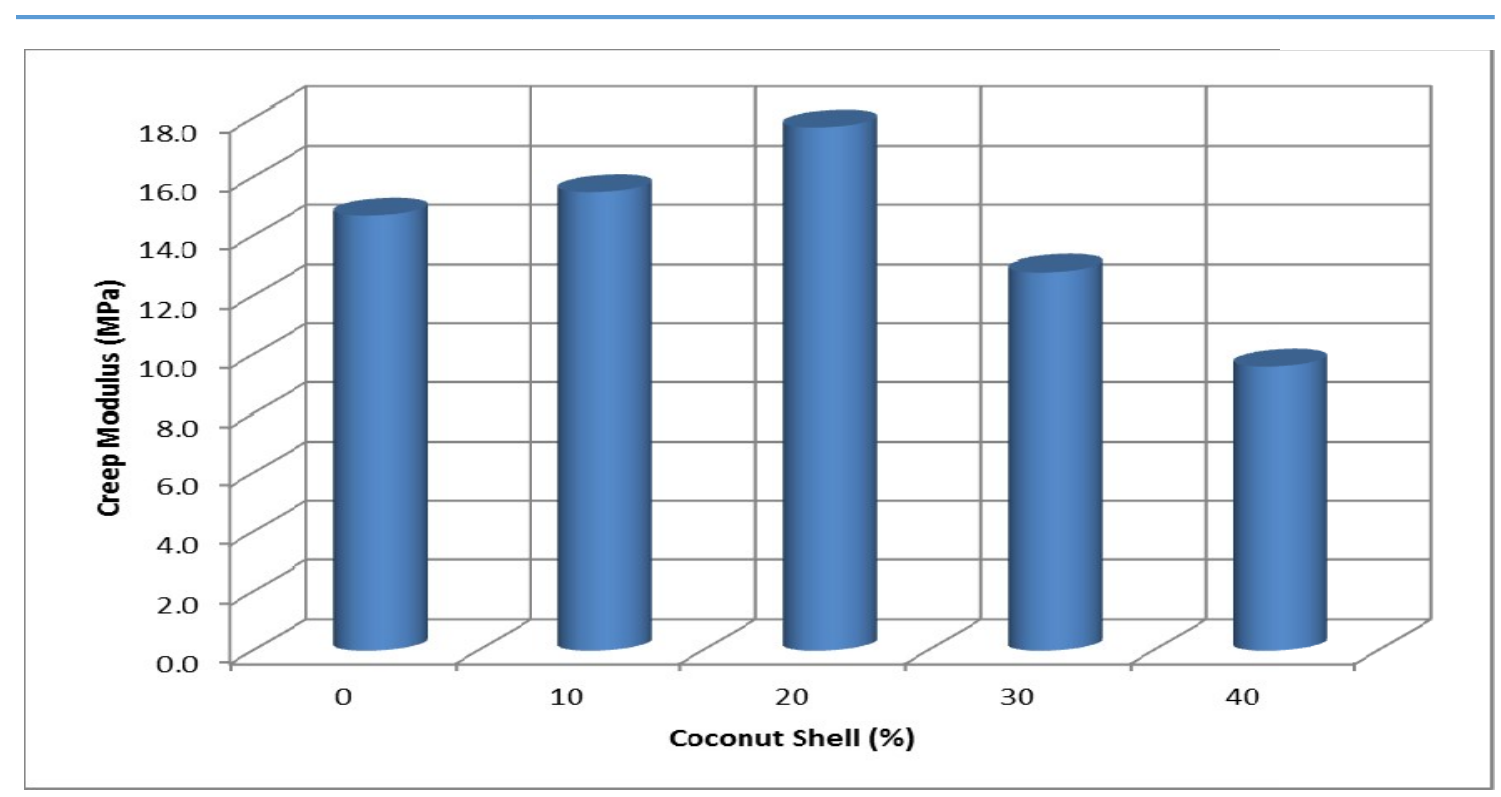

Fig.3. Dynamic creep of asphalt mixture at different percent CS

\subsection{Cumulative Strain and Time of Loading}

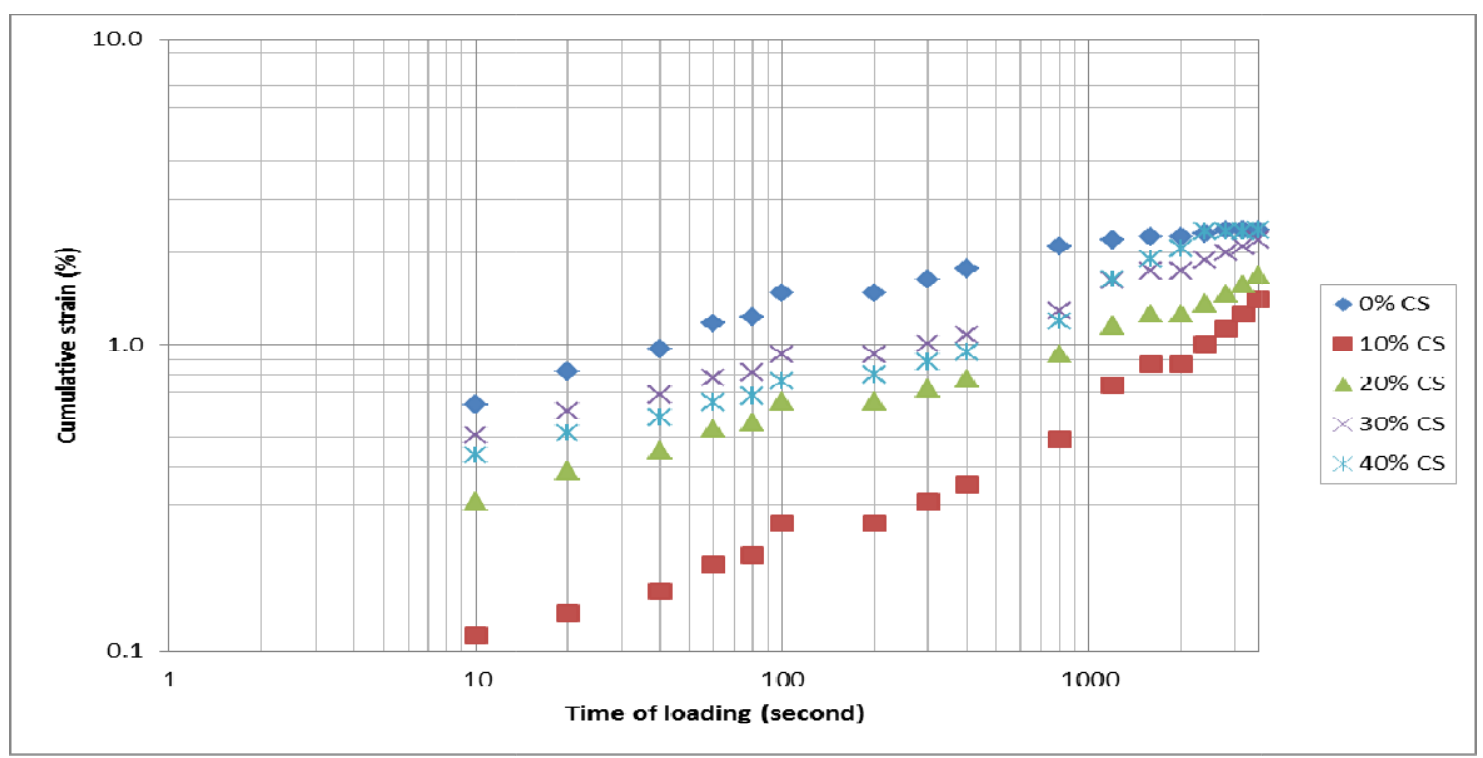

Fig.4. Accumulated strain versus time of loading

Fig. 4 presents the relationship between the logarithms of cumulative creep strain and loading time of specimens tested at different percentages of CS. The slope indicates the sensitivity of the mix to rutting. The resistance to the permanent deformation of a mix is expected to increase when the relative intercept becomes less, and the slope of the line decreases. The permanent deformation of the mixture containing $10 \% \mathrm{CS}$ with a strain value of $0.521 \%$ is slightly lower than those of other mixtures. In addition, the strain value of $0 \%, 20 \%, 30 \%$ and $40 \%$ CS is approximately $0.912,0.730,0.735$ and $0.825 \%$ respectively. Therefore, CS, as the 
aggregate replacement in the asphalt mixture, exhibits a better rutting resistance or less proneness to rutting compared with those of the original mixture.

\section{CONCLUSION}

The effects of CS on the stiffness modulus and creep properties were investigated. CS as an aggregate replacement material provides additional improvements in the resilient modulus and dynamic creep. The use of 10\%-20\% CS results in the good performance of modulus and creep stiffness.

\section{ACKNOWLEDGEMENTS}

The support provided by Malaysian Ministry of Higher Education and UniversitiTeknologi Malaysia in the form of a research grant vote no. Q.J130000.2522.14H30 and Q.J130000.2522.11H58 for this study is highly appreciated.

\section{REFERENCES}

[1] JusliE,NorH M,JayaRP,HaronZ. Strength and microstructure properties of double layered concrete paving blocks containing waste tyre rubber granules. JurnalTeknologi, 2015, 73(4):85-90

[2] Mohd YM I,Putra JR, HaininM R,IsmailC R,Wan IM H. Strength of porous concrete pavement at different curing methods. JurnalTeknologi, 2015, 76(14):99-103

[3] HidayahN A H, HasananM,RamadhansyahP J. Effect of coarse aggregate sizes on properties of porous concrete paving blocks. Advanced Materials Research, 2014, 911:433-437

[4] JusliE,Md NH,RamadhansyahP J,ZaitonH. Double layer concrete paving blocks using waste tyre rubber as aggregate replacement.Applied Mechanics and Materials, 2014, 554:128-132

[5] TarmuziN A, JayaR P,YaacobH,HassanN A, AzizM MA. Aggregate angularity effect on porous asphalt engineering properties and performance. JurnalTeknologi, 2015, 73(4):99-104 
[6] OlanipekunE A, OlusolaK O, Ata O. A comparative study of concrete properties using coconut shell and palm kernel shell as coarse aggregates. Building and Environment, 2006, 41(3):297-301

[7] NorhafizahM, RamadhansyahP J, SitiNur AJ, Nurfatin AM, NorhidayahA H, HaininM R, Che NC W. The effect of coconut shell on engineering properties of porous asphalt mixture. JurnalTeknologi, 2016, 78(7-2):127-132

[8] Ma B, Adhikari S, Chang Y, Ren J, Liu J, You Z. Preparation of composite shape-stabilized phase change materials for highway pavements. Construction and Building Materials, 2013, 42:114-121

[9] RamadhansyahP J, Nurfatin AM, Siti N AJ, NorhafizahM, NorhidayahA H, DewiS J. Use of coconut shell from agriculture waste as fine aggregate in asphaltic concrete. ARPN Journal of Engineering and Applied Sciences, 2016, 11(12):7457-7462

[10]Ali M, Liu A, Sou H, Chouw N. Mechanical and dynamic properties of coconut fibre reinforced concrete. Construction and Building Materials, 2012, 30:814-825

[11]PrustyJ K, PatroS K. Properties of fresh and hardened concrete using agro-waste as partial replacement of coarse aggregate-A review. Construction and Building Materials, 2015, $82: 101-113$

[12]HassanN A, MahmudM, JayaR P.Air void characterisation in porous asphalt using x-ray computed tomography. Advanced Materials Research, 2014, 911:443-448

[13]TingT L, JayaR P, HassanN A, Yaacob H, JayantiD S, AriffinM A M. A review of chemical and physical properties of coconut shell in asphalt mixture. JurnalTeknologi,2016, 78(4):85-89

[14]JeffryS N A, JayaR P, ManapN, MironN A, HassanN A.The influence of coconut shell as coarse aggregates in asphalt mixture. Key Engineering Materials, 2016, 700:227-237

[15]Malaysian Public Works Department (JKR). JKR/SPJ/2008-S4. Standard specification for road works. Kuala Lumpur: JKR, 2008

[16]American Society for Testing and Materials (ASTM). ASTM D7369-11: Standard test method for determining the resilient modulus of bituminous mixtures by indirect tension test. Philadelphia: ASTM International, 2011 
[17]British Standards Institution (BSI). BS EN 12697-25:2016. Bituminous mixtures. Test methods. Cyclic compression test. London: BSI, 2016

\section{How to cite this article:}

Yaacob H, Putra Jaya R, Madzaili A.H, Abdul Hassan N, Abdullah M.E, and Jayanti D.S. Stiffness Modulus and Creep Properties of the Coconut Shell in an Asphalt Mixture. J. Fundam. Appl. Sci., 2017, 9(6S), 50-58. 